\title{
Aspectos da reprodução e dinâmica populacional de Chione pubera (Bory Saint-Vicent) (Bivalvia, Veneridae) no sul do Brasil
}

\author{
Carlos Alberto Borzone ${ }^{1}$ \\ Kelly Midori Vargas ${ }^{1}$ \\ Paulo Ricardo Pezzuto ${ }^{2}$ \\ Yara Aparecida Garcia Tavares ${ }^{1}$
}

\begin{abstract}
General features of the reproduction and population dynamics of Chione pubera (Bory Saint-Vicent) (Bivalvia, Veneridae) in South Brazil. Material collected with a 2-meter wide beam trawl during eight surveys conducted from December 1995 to February 1997 was used for a first preliminary description of the reproduction and population dynamics of the edible venerid bivalve Chione pubera (Bory Saint-Vicent, 1827). Greatest catches were found between 30 to 35 meters depth, on fine sandy bottoms with a mud content lower than $10 \%$. Dry weight meat condition index showed highest values during autumn and lowest values from winter to summer months. Histological analysis of gonadal tissue showed a continuous reproduction, with peaks of maturation on autumn and spring. Spawning was related with the increase of water temperature, that occurs twice a year in the region as a consequence of a particular hydrological characteristic of the inner continental shelf. Size frequency distribution showed a unique principal mode with slow if any displacement through time, indicating a long lived species.
\end{abstract}

KEY WORDS. Chione pubera, reproduction, venerid, south Brazil

A família Veneridae reúne um grupo de bivalves muito apreciados pelo delicado sabor da sua carne. Muitas espécies são extensivamente pescadas em diferentes costas do mundo, tendo sido desenvolvido também com sucesso o cultivo das mesmas. Um exemplo bem conhecido é o de Mercenaria mercenaria (Linnaeus, 1758) no Atlântico norte (CARRIKER 1959). Esta espécie representa um importante recurso pesqueiro, existindo numerosos estudos sobre a sua biologia e ecologia populacional, constituindo hoje um dos venerideos melhor estudados (LOOSANOFF 1937; CARRIKER 1961; ANSELl 1963, 1964; ANSELl \& LOOSMORE 1963; ANSELL et al. 1964a,b; PORTER 1964; KEMISH 1980; MANZI et al. 1985).

Em outras costas do mundo não faltam exemplos de pescarias desenvolvidas sobre estes bivalves. Na Europa, Venus verrucosa (Linnaeus, 1758) e Callista chione (Linnaeus, 1758) foram intensamente pescadas, atingindo a primeira uma extração anual de 3.500 toneladas em 1980 no Mar do Norte (BERTHOU \& LE-GALL

1) Centro de Estudos do Mar, Universidade Federal do Paraná. Avenida Beira Mar, Pontal do do Sul, 83255-000 Pontal do Paraná, Paraná, Brasil.

2) Centro de Ciências Tecnológicas, da Terra e do Mar, Universidade do Vale do Itajaí. Caixa Postal 360, 88302-202 Itajai, Santa Catarina, Brasil. 
1980; BERTHOU et al. 1980). No golfo de Trieste, as pescarias de venerídeos tiveram um grande desenvolvimento desde a década dos anos 70. As primeiras espécies pescadas foram as de distribuição mais costeira, como Ensis minor (Linnaeus, 1758) e Chamelea gallina (Linnaeus, 1758). Com a diminuição das populações destas espécies, os pescadores procuraram outras alternativas em áreas mais profundas, iniciando a exploração de Venus verrucosa e Callista chione, venerideos que tiveram um alto valor no mercado internacional (DEL-PIERO 1994).

Na América Latina, existem pescarias importantes no México, como a "almeja chocolate", Tivela stultorum (Mawe, 1823) (CARRIQUIRIBORDE et al. 1981), a "almeja negra", Chione fluctifraga (Sowerby, 1853) (MARTINEZ-CORDOVA 1988), Chione undatella (Sowerby, 1853) (BAKEIRO-CARDENAS \& MASSO-ROJAS 1988) e Chione californienses (Broderip, 1853) (GARCIA-DOMINGUEZ et al. 1993; Castro-Ortiz \& Garcia Dominguez 1993; Garcia Dominguez et al. 1994); no Chile e no sul da Argentina, várias espécies de venerideos são intensamente pescadas, sendo Protothaca thaca (Molina, 1782) e Venus antiqua (King \& Broderip, 1835) as espécies melhor conhecidas (LYNCH \& MONARDEZ 1983; Osorio et al. 1983, 1987; LOZADA \& Bustos 1984; BORZONE 1992)

No Brasil, apesar da existência de uma variada fauna de moluscos representada por muitas espécies potencialmente exploráveis, poucas têm sido aproveitadas. Dentre os venerideos, apenas o pequeno "berbigão", Anomalocardia brasiliana (Gmelin, 1791) está sujeito a uma exploração comercial incipiente (NARCHI 1972; Pezzuto \& EChTERnacht 1999). Dentre os diferentes representantes do gênero Chione Muhlfeld, 1811, C. pubera (Bory Saint-Vicent, 1827) é a espécie de maior tamanho, com adultos medindo acima dos $7 \mathrm{~cm}$ de comprimento. Esta espécie se distribui desde o Rio de Janeiro até Santa Catarina (Rios 1994), sendo uma das espécies numericamente dominantes nos fundos de areia fina entre 20 e 50 metros de profundidade, na plataforma continental da costa sudeste do Brasil (GONÇALVES \& LANA 1991).

Um cruzeiro de prospecção de vieiras feito entre os dias 5 e 9 de novembro de 1995 pelo N/Pq. Diadorim do CEPSUL/IBAMA, mostrou a existência de um importante banco de C. pubera na frente do litoral sul do estado de São Paulo, a profundidades de 35 metros. A captura deste venerídeo superou amplamente à da vieira Euvola ziczac (Linnaeus, 1758) (anteriormente Pecten ziczac) (aproximadamente 350 indivíduos de C. pubera contra 20 de E. ziczac, por hora de arrasto) (Borzone \& PEZzUTo 1997). Embora os cruzeiros posteriores não tenham sido orientados para o estudo do venerídeo, foi possível ao longo de um ano obter o material necessário para realizar uma descrição preliminar da reprodução e da dinâmica populacional da espécie, cujos principais aspectos são apresentados na presente contribuição.

\section{MATERIAL E MÉTODOS}

O material estudado foi obtido em oito cruzeiros oceanográficos realizados na plataforma continental interna da região sudeste-sul, a bordo do N/Pq. Diadorim do CEPSUL/IBAMA. O primeiro cruzeiro de prospecção foi feito entre os dias 4 e 9 de dezembro de 1995 e compreendeu uma rede de 28 estações distribuídas em 
sete transversais entre as isóbatas de 20 e 50 metros (Fig. 3). Num segundo cruzeiro de prospecção feito entre os dias 20 e 22 de abril de 1996, foram estabelecidas 15 estações de amostragem limitadas ao banco de Chione pubera na localidade de Bom Abrigo. Os cruzeiros restantes para a amostragem da espécie foram feitos apenas neste último local, entre as coordenadas de $25^{\circ} 29^{\prime} 062^{\prime \prime}$ e $25^{\circ} 28^{\prime} 127^{\prime \prime} S$ e $47^{\circ} 37^{\prime} 317^{\prime \prime}$ e 4736'370"W. Cada estação de amostragem foi posicionada por meio de navegador GPS e a profundidade registrada em ecossonda. Amostras de sedimento e perfis oceanográficos foram utilizados para desenvolver uma caracterização oceanográfica da área de estudo (Borzone et al. 1999).

$\mathrm{Na}$ obtenção do material biológico foi utilizada uma rede de arrasto do tipo beam-trawl, com marco metálico medindo $1,90 \times 0,45 \mathrm{~m}$, rede de $5 \mathrm{~cm}$ de malha e corrente espantadeira de $9 \mathrm{~mm}$. Apenas no primeiro cruzeiro foi testado em estações complementares, o mesmo beam-trawl mas com uma corrente espantadeira de 19 $\mathrm{mm}$. No segundo cruzeiro de prospecção foi testada uma draga de arrasto de $0,80 \mathrm{x}$ 0,32 metros de embocadura e rede de $5 \mathrm{~cm}$, e realizado um mergulho autônomo para observação e filmagem submarina. Informação detalhada destes cruzeiros pode ser consultada em Borzone \& Pezzuto (1997), Pezzuto \& Borzone (1997a) e Pezzuto et al. (1998).

Todo o material de Chione pubera obtido nos diferentes cruzeiros foi medido no seu comprimento ântero-posterior com um paquímetro (precisão de $0,02 \mathrm{~mm}$ ) e teve as suas conchas guardadas. $\mathrm{Na}$ análise do índice de condição, aproximadamente 25 indivíduos de cada cruzeiro (abril de 1996, maio de 1996, junho de 1996, julho de 1996, agosto de 1996, outubro de 1996, dezembro 1996 e fevereiro de 1997) foram mensurados e após escorrido o líquido intravalvar, pesados em balança digital (precisão de $0,001 \mathrm{~g}$ ) para obtenção do peso úmido total (PUt). As carnes foram separadas e pesadas novamente para obtenção do peso úmido da carne (PUcr). Concha e carne separadas foram posteriormente secadas em estufa a $80^{\circ} \mathrm{C}$ por 30 horas obtendo-se assim o peso seco da conchaPSch e peso seco da carne-PScr. Foi obtido para cada indivíduo, um índice de condição para o peso úmido (ICu) e um índice de condição para o peso seco (ICs), segundo a relação:

$$
\mathrm{ICu}=(\mathrm{PUcr} / \mathrm{PUt}) * 100 \mathrm{e} \mathrm{ICs}=(\mathrm{PScr} /(\mathrm{PSch}+\mathrm{PScr})) * 100
$$

Para a análise histológica do ciclo reprodutivo, um número variado (9 a 20) de indivíduos adultos (acima de $50 \mathrm{~mm}$ ) de cada um dos cruzeiros mencionados, foi fixado em formalina $10 \%$ imediatamente após a sua obtenção em campo. Uma secção transversal do tecido gonadal de cada indivíduo foi processada em uma série crescente de desidratação, diafanizada, incluída em parafina, cortada com micrótomo manual do tipo Minot com espessura de $7 \mu \mathrm{m}$, sendo os cortes corados com a coloração bicrômica usual de Hematoxilina-Eosina. As lâminas foram analisadas em microscópio óptico para a verificação do grau de desenvolvimento da série gametogênica e demais aspectos estruturais histológicos. Nas fêmeas foram medidos os diâmetros do folículo e dos ovócitos com ocular micrométrica, e contados o número de folículos por meio campo visual da ocular do microscópio, além do número de ovócitos por folículo. Os exemplares analisados foram agrupados em estágios segundo a maturação das gônadas. 
As comparações das médias dos índices de condição entre os diferentes cruzeiros foram feitas através de uma análise de covariância (ANCOVA), considerando o comprimento dos indivíduos como uma covariável e os diferentes cruzeiros como fator de variação. Foi utilizado como teste a posteriori o de Diferença Significativa Mínima - MDS (SNEDECOR \& COCHRAN 1967), expressando seus resultados como gráficos de médias.

\section{RESULTADOS}

\section{Área de estudo}

Hidrologicamente, toda a região a profundidades maiores dos vinte metros sofre efeitos da intrusão de uma massa de água denominada Água Central do Atlântico Sul (ACAS), por baixo das águas costeiras dominadas pela Corrente do Brasil. No verão observa-se a intrusão destas águas frias e ricas em nutrientes sobre grandes extensões da plataforma continental, resultando na formação de uma forte termoclina aproximadamente de novembro a abril (MATSUURA 1986; Castro Filho 1990; Borzone et al. 1999). Durante o inverno, a presença de águas frias e de baixa salinidade é causada pela intrusão de águas de origem subantártica (CAMPOS et al. 1996; BORZONE et al. 1999), resultando num forte ciclo térmico semi-anual (Fig. 1). De acordo com Borzone et al. (1999), os sedimentos na área estudada apresentaram-se relativamente homogêneos, com as poucas variações sempre relacionadas ao gradiente batimétrico. Predominaram areias quartzosas finas a muito finas $(2,70$ a 3,27 $\phi)$, com frações de silte-argila aumentando com a profundidade, porém nunca excedendo os $10 \%$. O grau de seleção do sedimento foi moderado $(<0,9)$, e os valores de curtose apresentaramse altos $(>8)$ ao sul da área estudada e em profundidades intermediárias (30-40 $\mathrm{m})$. Os teores de matéria orgânica e de carbonato de cálcio foram baixos $(<3,5 \mathrm{e}$ $<12,22 \%$, respectivamente) e mostraram sempre um aumento com a profundidade. Ao norte da área, e nas estações mais próximas da costa, os valores $\phi$ do diâmetro médio do sedimento diminuíram indicando grãos mais grosseiros (Fig. 2) (BORZONE et al. 1999).

\section{Distribuição de Chione pubera}

C. pubera esteve presente em praticamente todas as estações amostradas no primeiro cruzeiro de prospecção, mas foi mais abundante nas estações mais rasas e ao norte da Baía de Paranaguá. Nesta área, as maiores capturas (acima de 59 individuos/hora de arrasto) ocorreram entre os 30 e os 35 metros de profundidade (Fig. 3). Todos os cruzeiros mostraram a diminuição ou desaparecimento do venerídeo a profundidades maiores que 45 metros. Importantes variações na sua captura foram registradas quando empregados diversos amostradores (Tab. I). A draga e o beam-trawl com corrente espantadeira de $19 \mathrm{~mm}$ de bitola, mostraram as maiores densidades, em resposta a uma maior penetração no sedimento. Contudo, as densidades podem ser consideradas baixas, já que se atribuirmos à draga uma eficiência máxima de $50 \%$, as densidades médias do venerídeo não superariam os 0,14 indivíduos por $\mathrm{m}^{2}$. 

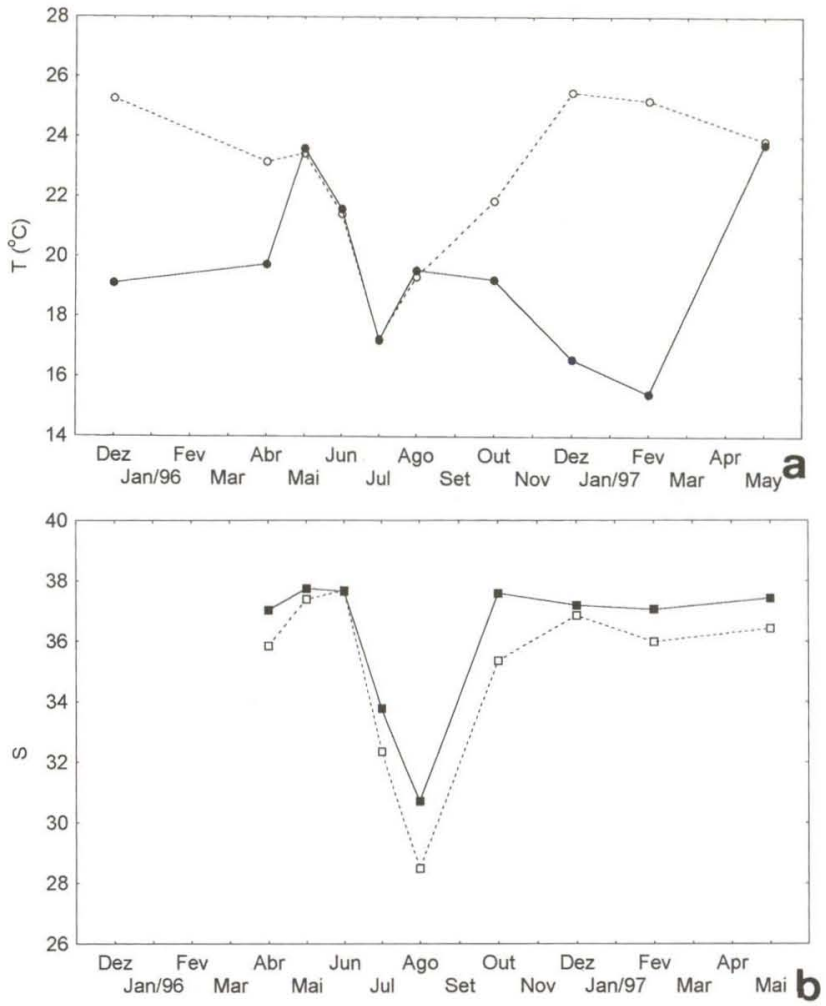

Fig. 1. Variações da temperatura (a) e da salinidade (b) de superfície (circulos brancos, linha tracejada) e de fundo (circulos pretos, linha inteira) na estação de amostragem de Chione pubera.

Tabela I. Diferentes amostradores e capturas obtidas de Chione pubera para uma mesma estação de amostragem $\left(25^{\circ} 28,735 \mathrm{~S}\right.$ e $\left.47^{\circ} 37,621 \mathrm{~W}\right)$ a $39 \mathrm{~m}$ de profundidade.

\begin{tabular}{lccc}
\hline \multicolumn{1}{c}{ Amostrador } & Indivíduos capturados & $\begin{array}{c}\text { Área percorrida } \\
\left(\mathrm{m}^{-2}\right)\end{array}$ & $\begin{array}{c}\text { Densidade } \\
\left.\text { (ind. * } \mathrm{m}^{-2}\right)\end{array}$ \\
\hline Beam trawl - espantadeira $9 \mathrm{~mm}$ & 22 & 10260 & $0,22 * 10^{-2}$ \\
Beam trawl - espantadeira $19 \mathrm{~mm}$ & 212 & 3900 & $5,30 * 10^{-2}$ \\
Draga & 28 & 400 & $7,00 * 10^{-2}$ \\
\hline
\end{tabular}

\section{Índice de condição}

O índice de condição, expresso pela relação percentual entre o peso da carne e o peso total do organismo, não foi constante ao longo do tempo. A análise de covariância mostrou que existem diferenças significativas ao longo dos cruzeiros entre as médias dos índices de condição, tanto para o peso úmido quanto para o peso $\operatorname{seco}$ (Fig. 4). 

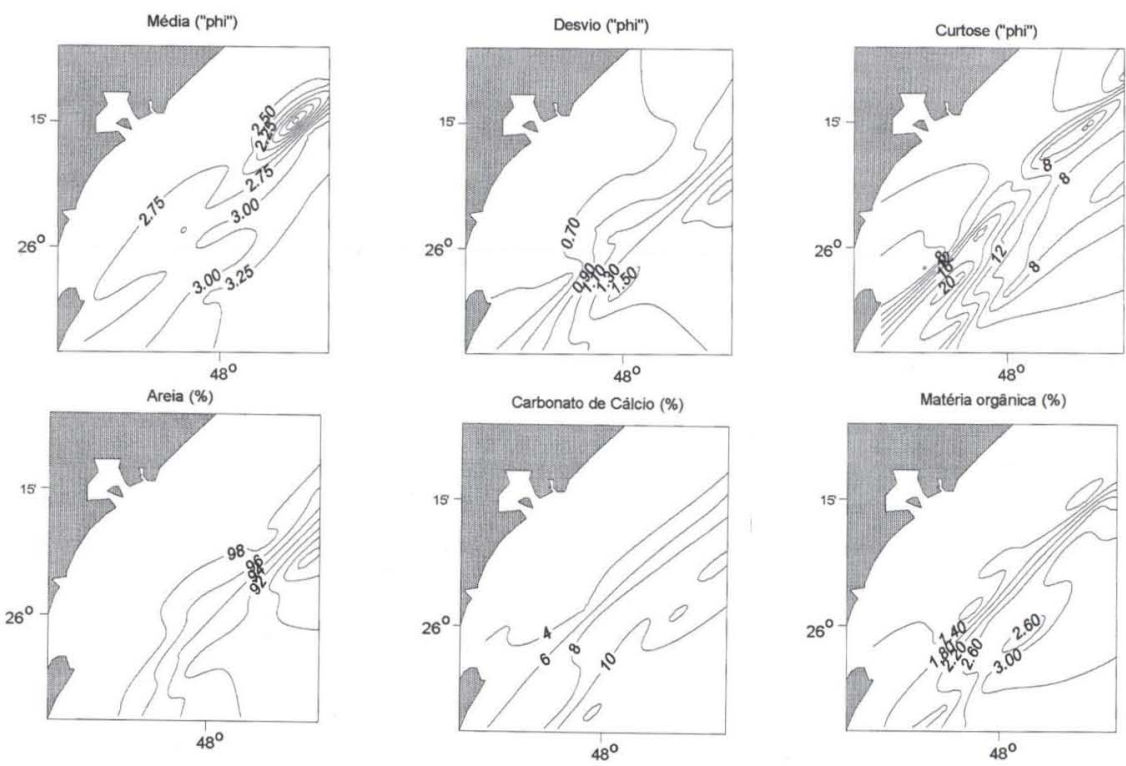

Fig. 2. Distribuição espacial de algumas variáveis sedimentológicas a partir de dados obtidos no primeiro cruzeiro de prospecção (4 a 9 de dezembro de 1995) (BORZONE et al. 1999).

O primeiro apresentou valores de média entre 21 e $22 \%$, existindo uma queda significativa do índice para 19,12\% no mês de agosto de 1996 . O valor máximo de fevereiro de 1997 de $22,05 \%$ não teve diferenças significativas com o restante dos meses (Fig. 4a). Já o índice de condição do peso seco mostrou valores altos no outono (de 4,73 a $5,00 \%$ ), com uma queda significativa no inverno e mantendo valores baixos nos meses de agosto, outubro e dezembro de 1996 (3,87, 4,03 e 3,84\%, respectivamente). O registro de fevereiro de 1997 mostra uma nova recuperação $(4,78 \%)$ (Fig. 4b).

\section{Analise histológica do ciclo reprodutivo}

As gônadas de C. pubera apresentam-se como um tecido que se estende entre os divertículos digestivos e o pé, e rodeando o tubo digestivo. Por este motivo, não é possível o reconhecimento macroscópico dos sexos sem uma dissecção do organismo. Os cortes histológicos mostraram que a espécie é dióica. Dos 117 exemplares analisados, 49 foram fềmeas $(41,88 \%)$ e 68 machos $(58,12 \%)$. A proporção sexual fêmea:macho variou mensalmente entre 1:1 e 1:2,2, sendo a média de 1:1,4 (Significativamente igual de $1: 1, X^{2}=3,0149 ; \mathrm{p}<0,81$ ). Em nenhum caso foi encontrada evidência de hermafroditismo.

Após uma primeira análise dos cortes histológicos foi definida uma escala de maturidade sexual distinta para cada sexo. Nas fêmeas foram reconhecidos os seguintes estágios:

Maturação máxima: os exemplares neste estágio caracterizam-se por apresentar folículos expandidos e cheios de ovócitos maduros prontos para serem 


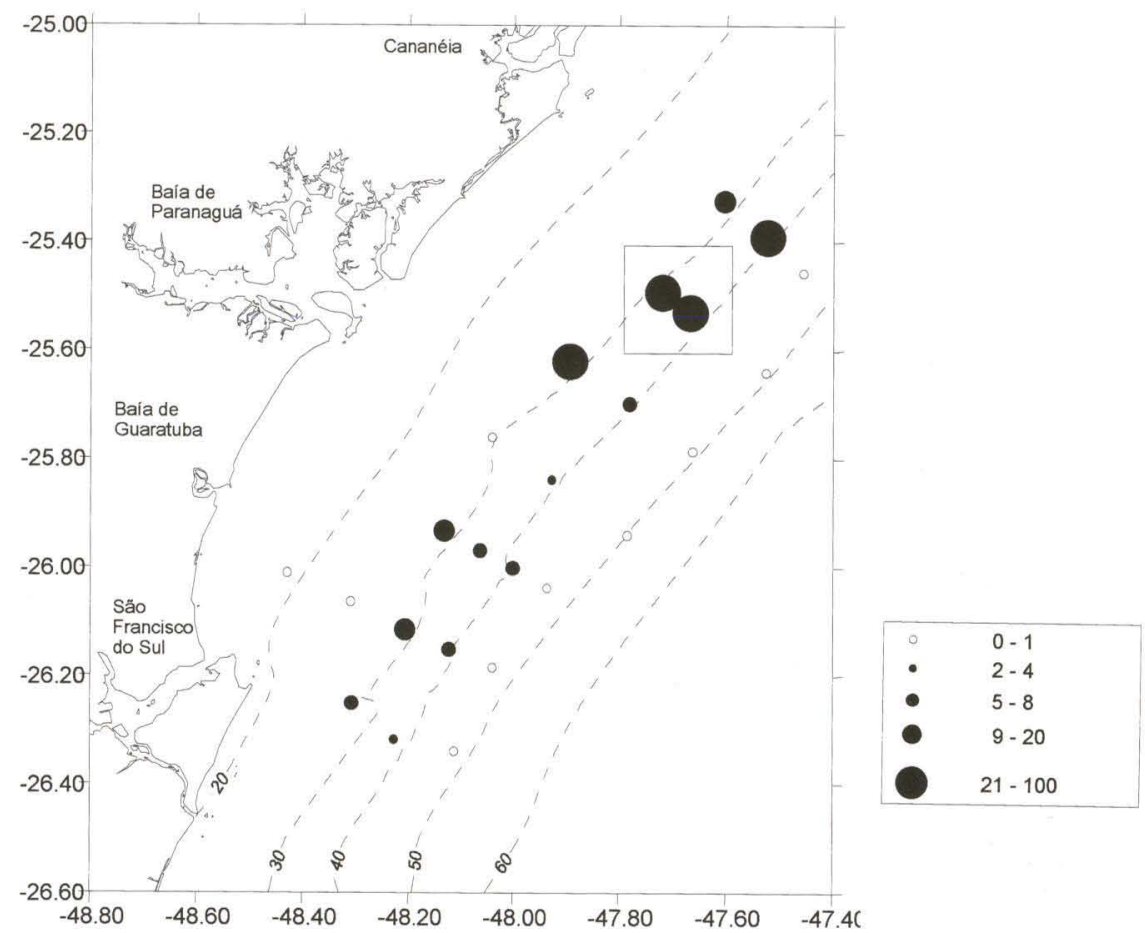

Fig. 3. Distribuição e densidade (em número de individuos por hora de arrasto) de Chione pubera a partir das coletas feitas no primeiro cruzeiro de prospecção (4 a 9 de dezembro de 1995). O quadro superior indica a área de maior densidade correspondente ao banco de Bom Abrigo.

expelidos (Fig. 5a). Muitos ovócitos apresentam formato poliédrico, devido à compressão exercida dentro do folículo. Existe pouco ou nenhum elemento proliferativo (células iniciais da série gametogênica, de coloração basófila). Os folículos possuem uma parede muito fina e quase não existe tecido interfolicular.

Desova parcial com proliferação: nesta fase ocorre uma desova juntamente com uma proliferação, o que indica a existência de uma desova contínua. A parede do folículo torna-se mais espessa. Os exemplares ainda apresentam células maduras residuais e ainda íntegras, no entanto aparecem muitos elementos proliferativos de vários tamanhos presos à parede do folículo (Fig. 5b).

Desova total com proliferação: praticamente todos os ovócitos maduros já foram liberados, levando a um completo esgotamento, porém os folículos permanecem íntegros nas suas formas originais. Não existe atividade fagocitária. A parede claramente torna-se mais espessa e muitos elementos proliferativos começam a aparecer aderidos à parede folicular (Fig. 5c).

Reversão: folículos de parede muito fina, apresentam-se com poucos ovócitos residuais no seu interior. Encontram-se muito reduzidos, tanto em número 

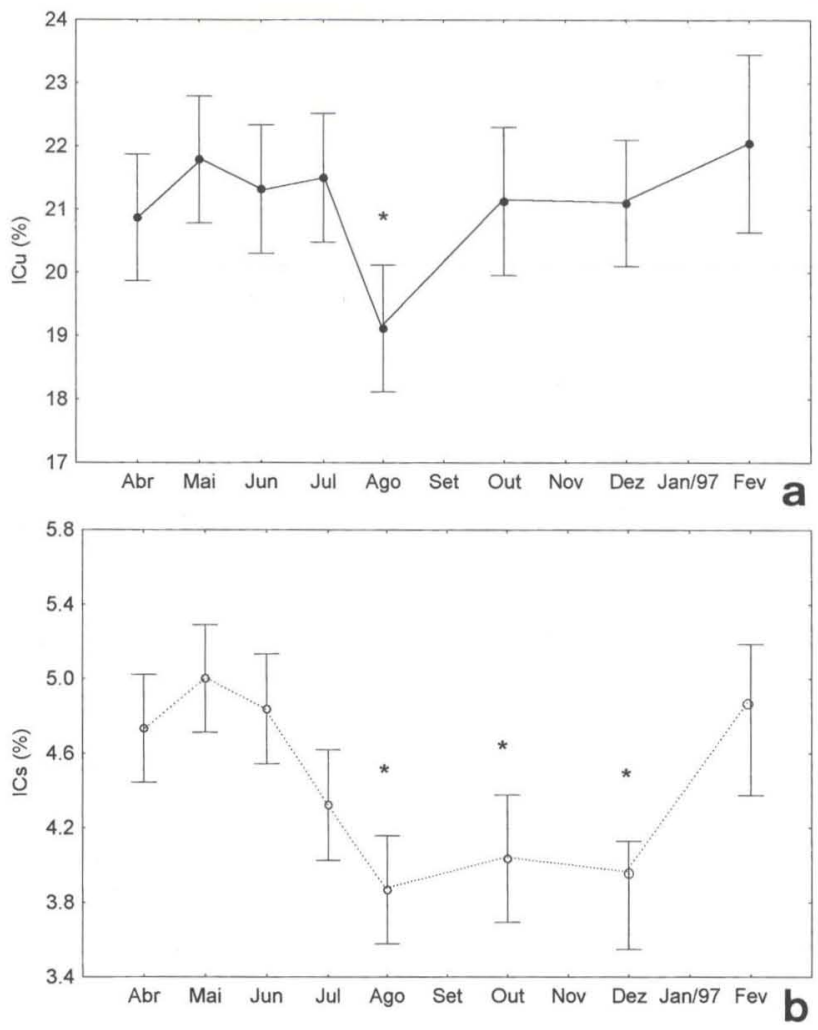

Fig. 4. Valores de média corrigida e intervalo de confiança $(95 \%)$ do indice de condição do peso úmido (a) e do peso seco (b). O asterisco indica diferenças siginificativas segundo resultados do ANCOVA (ICu: $F p=13,21, p<0,0001$; ICs: $F p=21,32, p<0,0001$ ).

quanto em tamanho (Fig. 5d). Ocorre um aumento do tecido interfolicular, onde é possível reconhecer um elevado número de amebócitos. Praticamente não existe atividade proliferativa. Os processos degenerativos são evidentes. Nesta fase, é provável que todo o «cido gonadal seja reabsorvido para serem formados novos folículos.

Nesta seqüência de estágios, após a maturação total dos folículos, um indivíduo pode desovar parcialmente, ao mesmo tempo em que ocorre uma proliferação ou desovar totalmente, sendo então seguida por uma proliferação ou uma reversão com reestruturação do tecido gonadal.

Em alguns indivíduos foi encontrado um tecido nutritivo associado à parede folicular, presente durante as fases de maturação máxima e ainda mais evidente durante a desova parcial com proliferação (Fig. 5b). Vários autores atribuem para este tecido a função de nutrir os gametas durante seu desenvolvimento e crescimento (LOOSANOFF 1937; PORTER 1964; QuAYle 1943).

Nos machos foram reconhecidos os seguintes cinco estágios:

Proliferação: presença de muitas células imaturas, de tamanho pequeno e uniforme, ocupando todo o espaço intrafolicular (Fig. 5e). Espermatogônias bem 
visíveis junto à parede dos folículos, os quais não apresentam a série espermática definida. A parede do folículo encontra-se mais espessa, assim como o tecido interfolicular.
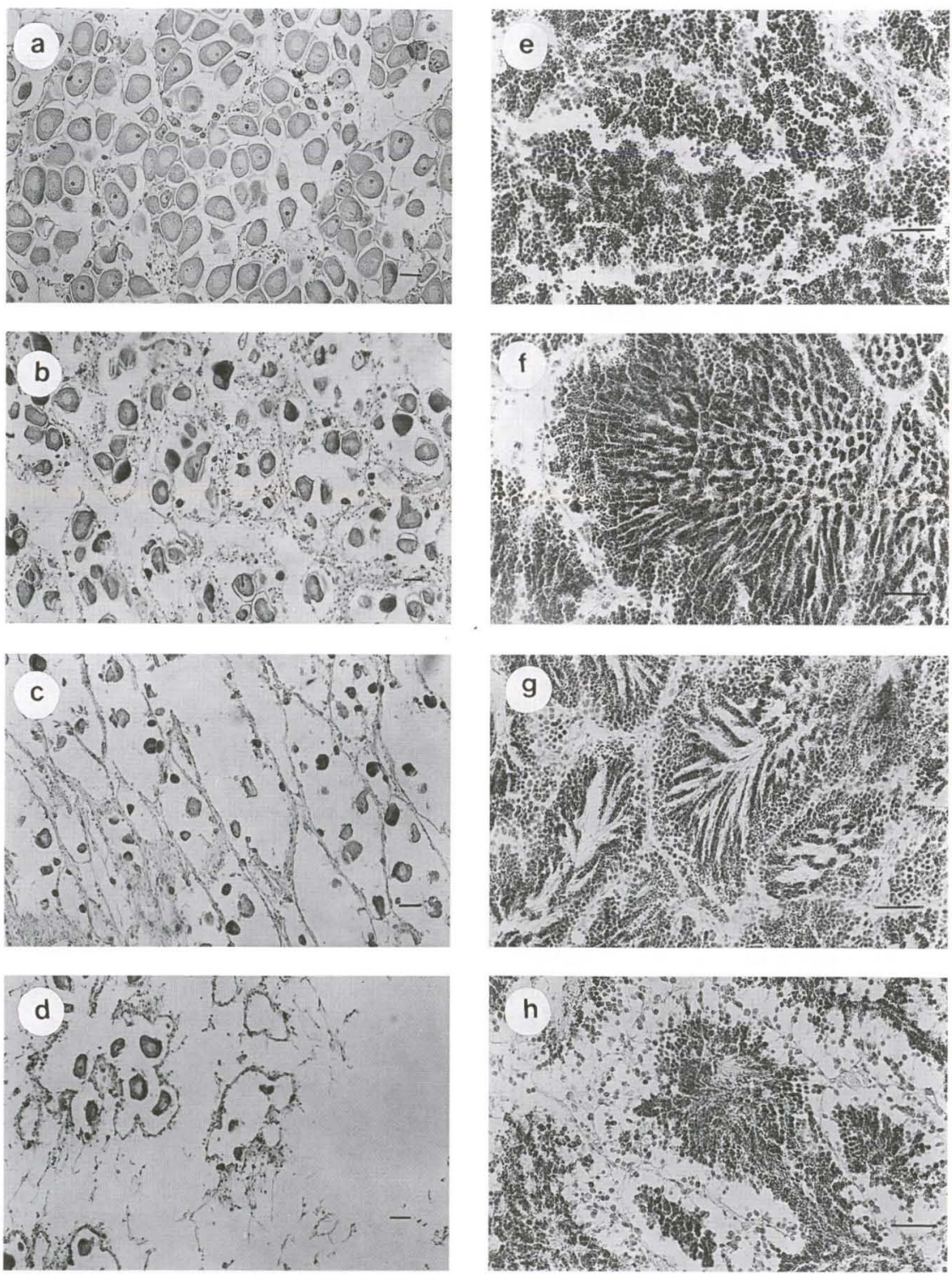

Fig. 5. Diferentes estágios de maturação em Chione pubera. Fêmeas: (a) Maturação máxima (b) desova parcial com proliferação, (c) desova total com proliferação, (d) reversão. Machos: (e) proliferação, (f) maturação máxima, (g) emissão com proliferação, (h) emissão com esgotamento. Escalas: $50 \mu \mathrm{m}$. 
Maturação máxima: a série espermática radial encontra-se bem desenvolvida, dominada por elementos completamente maduros (espermatozóides) ocupando toda a região central do lúmen formando pacotes ordenados (Fig. 5f). Espermatogônias e espermatócitos em número pequeno. A parede folicular encontra-se totalmente delgada e o tecido interfolicular está quase ausente. Alguns folículos já exibem um começo de desova reconhecível pelo desordenamento dos espermatozóides.

Emissão com proliferação: nesta fase ocorre uma emissão parcial de gametas, ou seja, os indivíduos ainda estão emitindo ao mesmo tempo que novos elementos proliferativos são formados, indicando um ciclo contínuo de produção de gametas. Observam-se claramente muitas células imaturas próximas à parede. Muitos espermatozóides ainda permanecem no lúmen (Fig. 5g).

Emissão com esgotamento: liberação progressiva dos gametas até o completo esvaziamento do folículo. A série espermática ainda está dominada por elementos maduros. Não existe proliferação e os elementos iniciais da série aparecem totalmente desprendidos da parede folicular (Fig. 5h). Os folículos permanecem integros nas suas formas, não havendo sinais de reabsorção, mas sim de engrossamento da parede folicular.

Reversão: como nas fêmeas, existe uma completa reabsorção do tecido gonadal e de todos os gametas residuais. Os folículos aparecem contraídos, com paredes rugosas e eventualmente quebradas, mostrando claros sinais de reversão. Diminuem em número e em tamanho, encontrando-se espalhados pelo tecido interfolicular dominante onde é visivel a presença de um número muito grande de amebócitos.

As freqüências dos estágios de maturação acima definidos mostram nas fêmeas a existência de uma desova parcial ao longo de todo o ano, com um parada da gametogênese nos meses de verão (Dez/96 e Fev/97) (Fig. 6a), evidenciado pelas imagens de reversão. Apesar de desovar de forma parcial praticamente durante todo o ano, dois picos de desova foram verificados, sendo o primeiro no outono, de abril, onde $71,4 \%$ dos indivíduos estavam em maturação total, a maio, quando $33,3 \%$ dos indivíduos estavam nesse mesmo estágio. O segundo pico de desova é o mais pronunciado e ocorreu na primavera, em outubro, correspondendo a $100 \%$ dos indivíduos em maturação total, sendo por isso considerada a desova mais importante. Em agosto, $50 \%$ dos indivíduos já estavam em maturação total.

Os machos também apresentam uma emissão contínua ao longo do ano e uma parada durante o verão (Fig. 6b). O pico de maturação máxima de outubro coincide com o das fêmeas, porém na emissão de abril existe uma grande percentagem de machos em estágio proliferativo. Isto determinaria um amadurecimento tardio dos espermatócitos, o que implicaria num atraso na liberação dos gametas masculinos, não coincidindo completamente com as fêmeas. É possível que o período em que as células proliferam e chegam até a maturação total para então serem liberadas é muito pequeno nos machos, suposição esta que também explicaria o grande número de indivíduos em esgotamento já no mês de maio.

A distribuição da freqüência do diâmetro dos ovócitos mostrou-se claramente bimodal nos meses de maio, agosto e outubro de 1996, indicando uma desova 

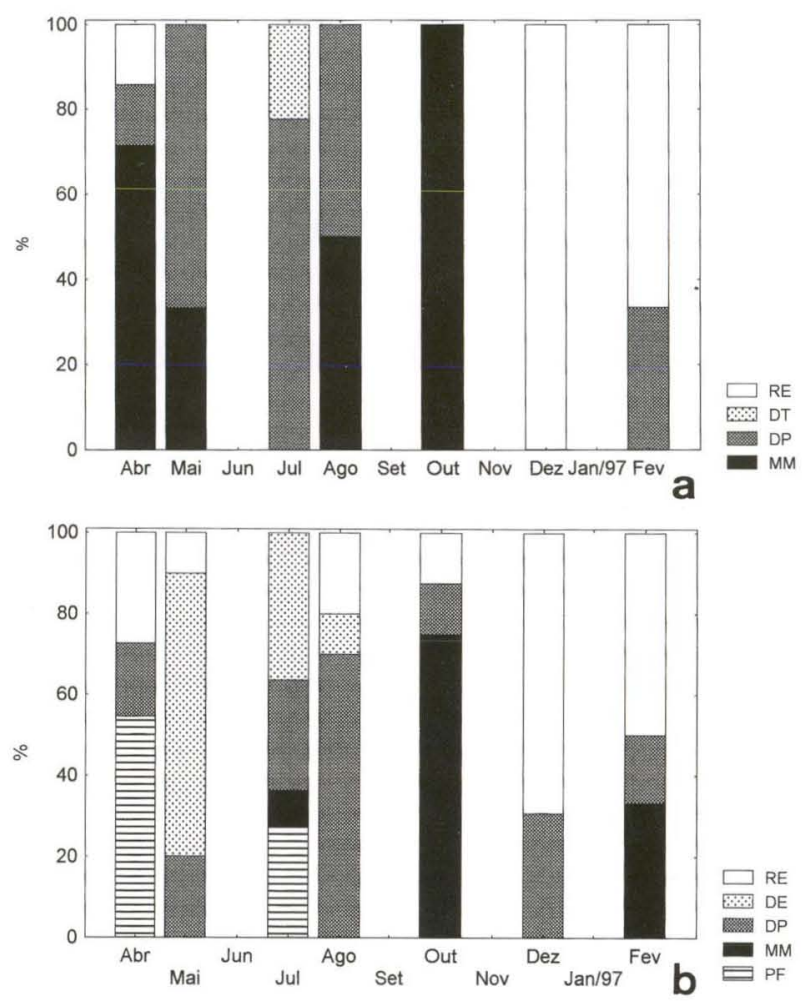

Fig. 6. Freqüência dos estágios de maturação em Chione pubera. (a) Fêmeas: (MM) maturação máxima, (DP) desova parcial com proliferação, (DT) desova total com proliferação, (RE) reversão; (b) Machos: (PF) proliferação, (MM) maturação máxima, (DP) desova com proliferação, (DE) desova com esgotamento, (RE) reversão.

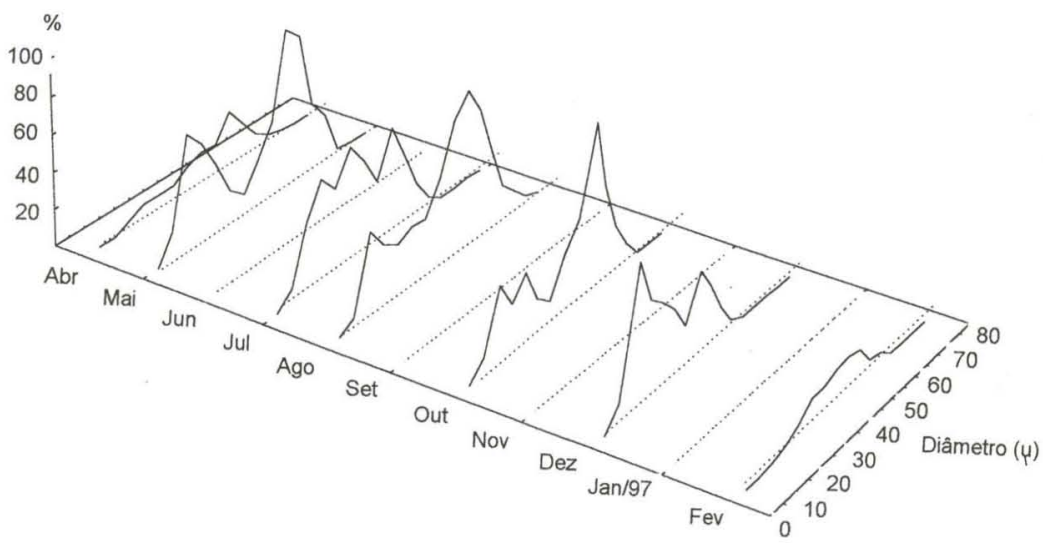

Fig. 7. Distribuição de freqüências de tamanho dos ovocitos em Chione pubera. 
contínua com proliferação em grande parte do ciclo anual estudado (Fig. 7). O mês de outubro, com a maior percentagem de indivíduos em maturação máxima, apresentou o maior número de ovócitos por folículo e, conseqüentemente, uma maior expansão destes últimos (Fig. 8). Em contraposição, um número pequeno de ovócitos por folículo e folículos menores foi registrado no primeiro pico de desova em abril, podendo ser esta relação um reflexo de pequenas desovas contínuas com recuperação e nova proliferação. O número de folículos mostrou-se inversamente proporcional ao seu diâmetro e este, proporcional ao número de ovócitos (Fig. 8).

a
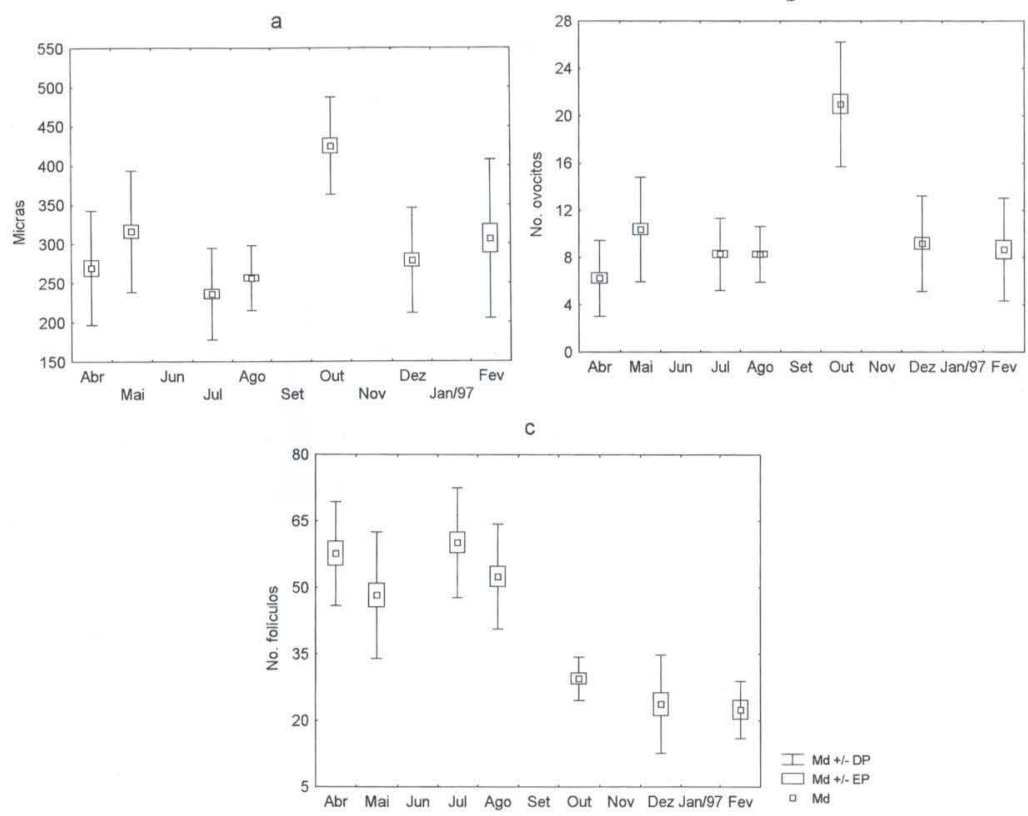

Fig. 8. Diâmetro máximo dos folículos (a), número de ovócitos por folículo (b) e número de folículos por campo de $5 \mathrm{~mm}^{2}$ (c) em Chione pubera.

\section{Analise da distribuição de comprimentos}

A variação temporal do comprimento das conchas mostrou uma moda principal em praticamente todas as amostras, predominando os indivíduos adultos de tamanho entre 60 e $70 \mathrm{~mm}$ (Fig. 9). Indivíduos menores foram amostrados em abril de 1996, no entanto com freqüências extremamente baixas impedindo desta forma quaisquer análises precisas da progressão modal e determinação do crescimento.

\section{DISCUSSÃO}

A existência de bancos de uma espécie de venerídeo com potencialidades para a comercialização representa uma informação inédita no conhecimento dos recursos marinhos vivos da plataforma interna do litoral sul brasileiro. Esta plata- 


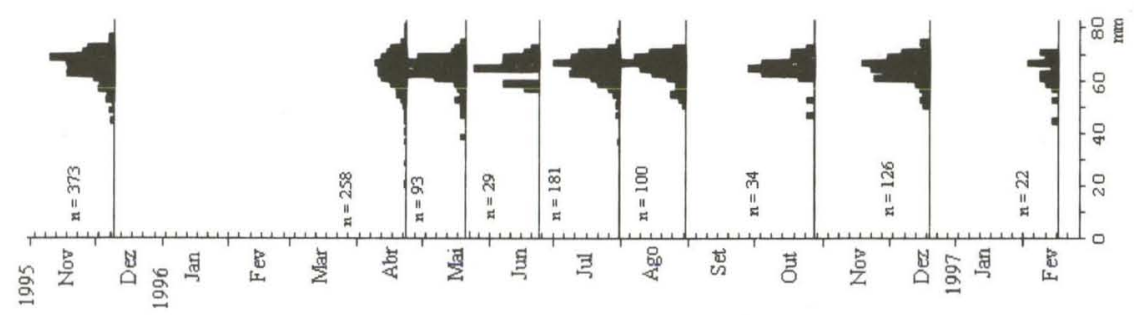

Fig. 9. Distribuiçăo de freqüências de comprimento em Chione pubera.

forma já foi cenário de uma das pescarias de bivalves mais importantes do Brasil, a da vieira Euvola ziczac (PEzzUTo \& Borzone 1997b). Embora as abundâncias estimadas para Chione pubera sejam extremamente baixas, mesmo quando testados equipamentos com maior penetração no sedimento, como a draga, deve-se considerar que estes equipamentos não são os mais adequados para uma espécie de bivalve infaunal que fica enterrada no sedimento em profundidades provavelmente maiores dos $10 \mathrm{~cm}$. Valores de abundância bem superiores poderiam ser obtidos se empregadas dragas comerciais hidráulicas nas amostragens, como as utilizadas na exploração de Macrocallista nimbosa (Lightfoot, 1786) na Flórida (Jolley 1972).

No presente levantamento, as maiores abundâncias de C. pubera mostraram estar relacionadas a um aumento do diâmetro médio do sedimento junto a valores de assimetria negativos (tendências aos grosseiros), os quais foram registrados ao norte da área de estudo e em estações mais rasas. A distribuição de freqüências de comprimento mostrou que a espécie, como muitos outros venerídeos, apresenta-se formando bancos com apenas uma classe de comprimento dominante. Isto é indicativo de um crescimento lento, sendo nestes casos indispensável a leitura de marcas ou anéis na concha para poder fazer uma estimativa do crescimento (LUTZ \& RHOADS 1980; ARNERI et al. 1998).

O estudo histológico da gametogênese revelou a existência de uma reprodução contínua, com picos no outono e primavera. Nas fêmeas, estas épocas são evidenciadas tanto na percentagem de indivíduos em estágios de maturação máxima e desova parcial com proliferação, quanto nas medidas de diferentes componentes estruturais e celulares da gônada. Estes últimos mostraram altos valores de diâmetro máximo de folículos coincidindo com alto número de ovócitos por folículo e baixo número de foliculos por campo. Esta relação foi máxima em outubro de 1996. No período de reversão gonadal, de dezembro a fevereiro, manteve-se uma relação de um número baixo de pequenos folículos por campo, o que indicaria também um aumento do tecido interfolicular, que segundo PIZARRO \& CRUZ (1987), seria inversamente proporcional ao grau de maturidade sexual.

Foi observado, embora de forma esporádica e apenas nas fềmeas, um tipo de tecido nutritivo que estaria associado às épocas de proliferação e maturação dos gametas. Segundo BORZONE (1992), este tecido apresenta um ciclo de desenvolvimento ao longo do ano, podendo estar relacionado aos aportes de nutrientes para o 
crescimento vitelogênico dos ovócitos. QUAYLE (1943) e PORTER (1964) encontraram este tecido também nos machos e o associaram com o desenvolvimento e crescimento dos gametas. LoOSANOFF (1937) curiosamente encontrou um tecido fagocítico-nutritivo em maior quantidade nos machos do que nas fêmeas. Nos machos este tecido forneceria nutrição para o desenvolvimento dos gametas, enquanto que nas fêmeas seu crescimento estaria associado à extração de nutrientes do mesênquima e de vasos sanguíneos entre os folículos. Em muitos casos, a presença deste tecido pode mascarar a interpretação reprodutiva das flutuações dos índices de condição.

Em C. pubera, o peso seco mostrou uma maior variação ao longo do ano do que o peso úmido, devido provavelmente à um maior acúmulo de água nos meses que apresentaram um baixo índice de condição para o peso seco. Este último manteve-se com valores altos durante o outono, máximo no mês de maio, e valores baixos durante a primavera, agosto a dezembro. Apesar do máximo de fêmeas maturas que ocorreu no mês de outubro, isso não esteve refletido no valor do índice que foi baixo. O mesmo ocorre em fevereiro, onde existiu uma alta percentagem de indivíduos em reversão gonadal, tanto em machos como em fềmeas, e o índice de condição apresentou um valor alto. Deve-se considerar que nos venerídeos as gônadas estão difundidas na base do pé e envolvendo o aparelho digestivo, e portanto não é possível a dissecção da gônada e a obtenção do peso da mesma. Desta forma, as variações do índice de condição não estarão necessariamente associadas aos eventos reprodutivos.

Chione pubera apresentou períodos de desova relacionados com o aumento da temperatura da água em profundidade. Por sua vez, o período de reversão gonadal tem coincidido com o registro de mínimos valores de temperatura em decorrência da incursão da ACAS (Água Central do Atlântico Sul). Os valores de máximos sub-superficiais de clorofila (MSC) alcançaram os maiores valores durante este período, nas camadas eufóticas mais profundas, ultrapassando os 30 metros de profundidade (PEZZUTO et al. 1998; BORZONE et al. 1999). Chama a atenção o fato de que em fevereiro, após este período de máxima entrada de nutrientes e alta produtividade, foi registrado um alto índice de condição. Tudo indica que, neste momento do ano, existiria um acúmulo de nutrientes fora do tecido gonadal, mas que teria como finalidade preparar os indivíduos para uma contínua atividade reprodutiva que se iniciaria no outono e se estenderia ao longo do ano até uma nova entrada da ACAS. As baixas temperaturas registradas em julho e agosto de $1996 \mathrm{e}$ associadas a intrusão de águas de origem subantártica (CAMPOS et al. 1996; BORZONE et al. 1999), parecem não afetar os processos da gametogênese, que são contínuos nesta época do ano, e que pelo contrário apresentam uma maturação máxima imediatamente após a intrusão desta água, na primavera. No entanto, existiria um forte esgotamento geral da condição dos indivíduos, o que se refletiria na queda dos índices de condição e a entrada numa fase de repouso ou reversão gonadal.

Existe uma grande semelhança entre os ciclos gametogênicos de outras espécies de venerideos, onde é comum o registro de reproduções contínuas. Chamelea striatula (da Costa, 1778) (ANSELl 1961), Protothaca staminea (Conrad, 
1837)(QUAYLE 1943), e Mercenaria mercenaria (PoRTER 1964; MANZI et al. 1985) exibem um disparo oogonial após as primeiras desovas, dando assim início a um longo período reprodutivo. Segundo LoOOSANOF (1937), a liberação de ovócitos maduros remove os fatores inibidores da produção e crescimento de uma nova séria de ovócitos. Quanto a relação com a temperatura da água, também parece ser comum no grupo que as desovas correspondam aos períodos de máxima temperatura. Por exemplo, Chione californiensis apresenta, no Pacífico norte, uma desova contínua durante quatro meses, com pico em agosto, coincidindo com a maior temperatura da água. BORZONE (1992) trabalhando com Venus antiqua no Atlântico sul, também descreveu um ciclo contínuo com maturações máximas na primavera e verão, e com uma breve fase de repouso ou reversão gonadal nos meses de inverno, coincidindo com a menor temperatura da água na área de estudo.

Os resultados apresentados neste trabalho mostram que o ciclo reprodutivo de Chione pubera ajusta-se a uma variação térmica semianual com redução da atividade reprodutiva nas estações com queda da temperatura da água (inverno e verão), seguindo desta forma os padrões que são, aparentemente, uma característica dos venerídeos.

AGRADECIMENTOS. Este trabalho recebeu apoio através de Bolsa de Demanda Social CAPES (PRP) e Bolsas de Pesquisa (CAB) e Iniciação Científica PIBIC (KMV) do CNPq.

\section{REFERÊNCIAS BIBLIOGRÁFICAS}

Ansell, A.D. 1961. The development of the primary gonad in Venus striatula (Da Costa). Proc. Mal. Soc. London 34 (5): 243-247.

-1963. The biology of Venus mercenaria in British waters and in relation to generating station effluents. Rep. Challenger Soc. 3 (15): 1-10.

- 1964. Some parameters of growth mature Venus mercenaria L. Jour. Conseil Int. L'Explor. Mer. 29 (2): 211-215.

ANSELL, A.D. \& F.A. LOOSMORE. 1963. Preliminary observations on the relationship between growth, spawning and condition in experimental colonies of Venus mercenaria L. Jour. Conseil Int. L'Explor. Mer. 28 (2): 285-294.

Ansell, A.D.; K.F. Lander; M. Coughlan \& F.A. LoOsmore. 1964a. Studies on the hard-shell clam, Venus mercenaria, in British waters. I. Growth and reproduction in natural and experimental colonies. Jour. Appl. Ecol. 1: 63-82.

Ansell, A.D.; F.A. Loosmore \& K.F. Lander. 1964b. Studies on the hard-shell calm, Venus mercenaria, in Brithish waters. II. Seasonal cycle in condition and biochemical composition. Jour. Appl. Ecol. 1: 83-95.

Arneri, E.; G. Giannetti \& B. Antolini. 1998. Age determination and growth of Venus verrucosa L. (Bivalvia: Veneridae) in the southern Adriatic and the Aegean Sea. Fish. Res. 38: 193-198.

BAKEIRO-CARdenAS, E. \& J.A. MASSO-Rojas. 1988. Variaciones poblacionales y reproducción de dos poblaciones de Chione undatella (Sowerby, 1835) bajo differentes regímenes de pesca en la Bahía de la Paz, B.C.S., Mexico. Cienc. Pesq. 6: 51-67.

Berthou, P. \& J.Y. Le-Gall. 1980. Selectivite de la drague a praires (Venus verrucosa, Mollusque, Veneridae) en Manche Occidentale. ICES, Copenhagen-Denmark, CM1980/B 11: 17.

Berthou, P.; J.Y. Le-Gal.; F. Diabal. \& M. Yahiadui. 1980. The clam Venus verrucosa (Mollusca, Veneridae) in the western English Channel and Normandy: Biology and fisheries. 1 Growth. ICES, Copenhagen-Denmark, CM1980/K 4: 36p.

Revta bras. Zool. 18 (2): 333 - 349, 2001 
Borzone, C.A. 1992. El ciclo gonadal de Venus antiqua King \& Broderip, 1835 (Bivalvia, Veneridae) en el golfo San José. Physis, Sec. A, 47 (113): 61-72.

Borzone, C.A. \& P.R. Pezzuto. 1997. Relatório técnico dos cruzeiros do Projeto Vieira. Cruzeiro I (4 a 9 de dezembro de 1995). Notas téc. FACIMAR 1: 67-79.

Borzone, C.A.; P.R. Pezzuto \& E. Marone. 1999. Oceanographic characteristics of a multi-specific fishing ground of the Central South Brazil Bight. Mar. Ecol. (P.S.Z.N.I) 20 (2): 131-146.

Campos, E.J.D.; Y. IKeda; B.M. Castro Filho; S.A. Gaeta; J.A. Lorenzzetti \& M.R. STE. 1996. Experiment studies circulation in the Western South Atlantic. Trans. Amer. geophys. Un. 77 (27): 253-259.

CARRIKER, M.R. 1959. The role of physical and biological factors in the culture of Crassostrea and Mercenaria in a salt-water pond. Ecol. Monogr. 29: 219-266.

- 1961. Interrelation of functional morphology, behavior and autoecology in early stages of the bivalve Mercenaria mercenaria. Jour. Elisha Mitchell scient. Soc. 77 (2): 168-242.

Carriquiriborde, L.; A. Farias; A. Gastelu \& O. Perez. 1981. Experimental transplant of Tivela stultorum Mawe in Baja California, Mexico. Cienc. Mar. 7 (1): 49-66.

CASTRo Filho, B.M. 1990. Estado atual do conhecimento dos processos fisicos das águas da plataforma continental sudeste do Brasil. In: ACIESP (Ed.), II Simposio de Ecossistemas da Costa Sul e Sudeste Brasileira, Lindóia, São Paulo, 4: 1-19.

Castro Ortiz, J.L. \& F. Garcia Dominguez. 1993. Crecimiento de Chione californiensis (Broderip, 1835) (Veneridae) en la Ensenada de La Paz, Baja California Sur, Mexico. Invest. Mar. CICIMAR $8(2): 45-52$.

Del-Piero, D. 1994. The clam fishery in the Gulf of Trieste. In: P.G. Wells \& P.J. Ricketts (Eds). Proc. Coastal zone Canada'94, Cooperation in the coastal zone 4: 1645-1660.

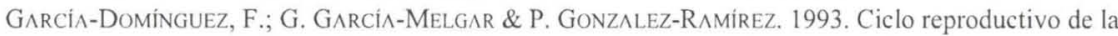
almeja ronosa, Chione californiensis (Broderip, 1835) en Bahia Magdalena, Baja California Sur, Mexico. Cienc. Mar. 19 (1): 15-28.

Garcia-DomingueZ, F.G.; S.A. Garcia-GasCA \& J.L. CASTro-OrTIZ. 1994. Spawning cycle of the red clam Megapitaria aurantiaca (Sowerby, 1831) (Veneridade) at Isla Espiritu Santo, Baja California Sur, Mexico. Jour. Shellfish Res. 13 (2): 417-423.

Gonçalves, E.M. \& P.C. LANA. 1991. Padrões de distribuição de bivalvia e gastropoda na plataforma continental da costa sudeste do Brasil $\left(24^{\circ} \mathrm{S}-27^{\circ} \mathrm{S}\right)$. Nerítica, Pontal do Paraná, 6 (1-2): 73-92.

JOLLEY, J.W. 1972. Exploratory fishing for the sunray venus clam, Macrocallista limbosa, in northwest Florida. Fla. Bd. Conserv. Mar. Res. Lab., Tech. Ser. 67: 42.

KEMISH, M.J. 1980. Shell microgrowth analysis. Mercenaria mercenaria as a type example for research in population dynamics, p. 255-292. In: D.C. RHOADS \& R.A. LUTZ (Eds) Skeletal growth of aquatic organisms. New York, Plenum Press, 750p.

LoOSANOFF, V.L. 1937. Seasonal gonadal changes of adult clams, Venus mercenaria (L.). Biol. Bull., Woods Hole, 72: 406-436.

Loz^d^, E. \& H. Bustos. 1984. Madurez sexual y fecundidad de Venus antiqua King \& Broderip, 1835, en la Bahia de Ancud (Mollusca-Bivalvia Veneridae). Rev. Biol. Mar., Valparaiso, 20 (2): 91-112.

LUTZ, R.A. \& D.C. RHOADS. 1980. Growth patterns within thw molluscan shell: an overview, p. 203-248. In: D.C. RHOADS \& R.A. LuTz (Eds). Skeletal growth of aquatic organisms. New York, Plenum Press, 750p.

LyNCH, J.Z. \& R.P. MonARDEZ. 1983. Estructura y crecimiento de una poblacion de Protothaca thaca (Molina, 1782). Estud. Oceanol. 3 (1): 75-82.

Manzi, J.J.; M.Y. Bobo \& V.G. Burrfil.. 1985. Gametogenesis in a population of the hard clam Mercenaria mercenaria (L) in North Santee Bay, South Carolina. The Veliger, 28 (2): 186-194.

Martinez-Córdova, L.R. 1988. Bioecologia de la almeja negra Chione fluctifraga (Sowerby, 1853). Rev. Biol. Trop. 36 (2A): 213-219.

MatsuURA, Y. 1986. Contribuição ao estudo da estrutura oceanográfica da região sudeste entre Cabo Frio (RJ) e o Cabo de Santa Marta Grande (RG). Ciência e Cultura, São Paulo, 38 (8): 1439-1450.

Revta bras. Zool. 18 (2): 333 - 349, 2001 
NARCHI, W. 1972. Comparative study of the functional morphology of Anomalocardia brasiliana (Gmelin, 1791) and Tivela mactroides (Born, 1778) (Bivalvia, Veneridae). Bull. mar. Sci., 22 (3): 643-670.

Osorio, C.; D. FrassinetTi \& E. Bustos. 1983. Taxonomia y morfometria de Venus antiqua King \& Broderip (Mollusca, Bivalvia, Veneridae). Tethys 11 (1): 49-56.

Osorio, R.C.; P.V. Bustos \& R.E. Bustos. 1987. Contenido gastrico de la almeja (Venus antiqua antiqua King \& Broderip, 1835) in Ancud, Chile (Mollusca: Veneridae). Invest. Pesq., Santiago, 34: 139-147.

Pezzuto, P.R. \& A.M. Echternacht. 1999. Avaliação de impactos da construção da Via Expressa SC-Sul sobre o berbigão Anomalocardia brasiliana (Gmelin, 1791) (Mollusca: Pelecypoda) na Reserva Extrativista Marinha do Pirajubaé (Florianópolis, SC - Brasil). Atlântica, Rio Grande, 21: 105-119.

Pezzuto, P.R. \& C.A. Borzone. 1997a. Relatório técnico dos cruzeiros do Projeto Vieira. Cruzeiro II (15 a 17 de março de 1996) e III (20 a 22 de abril de 1996). Notas Téc. FACIMAR 1: 67-79.

_ 1997b. The scallop Pecten ziczac (L., 1758) fishery in Brazil. Jour. Shellfish Res. 16 (2): 527-532.

Pezzuto, P.R.; C.A. Borzone; R.L.B.E. Abrahão; F. Brandini \& E.C. Machado. 1998. Relatório técnico dos cruzeiros do projeto vieira. III. Cruzeiros IV (maio de 1996) a XIV (maio de 1997). Notas Téc. FACIMAR, Itajaí, 2: 109-129.

Pizarro, J.F. \& R.A. Cruz. 1987. Ciclo reproductivo de la almeja Protothaca grata (Pelecypoda: Veneridae). Brenesia, San José, 27: 23-34.

Porter, H.J. 1964. Seasonal gonadal changes of adult clams Mercenaria mercenaria. Proc. Nat. Shellfish Ass. 55: 35-53.

QuAyle, P.B. 1943. Sex, gonad development and seasonal gonad changes in Paphia staminea (Conrad). Jour. Fish. Res. Bd. Can. 6 (2): 140-151.

Rıos, E. 1994. Seashells of Brazil. Rio Grande, Fund. Univ. Fed. Rio Grande, 2a ed., 492p.

Snedecor, G.W. \& W.G. Cochiran. 1967. Statistical methods. Iowa Iowa State Univ. Press, 703p.

Recebido em 16.III.2000; aceito em 24.IV.2001. 